\title{
Espectros de emisión de radioluminiscencia y termoluminiscencia de una leucita de Monte Somma (Italia)
}

\author{
V. CORRECHER', F.J. VALLE-FUENTES ${ }^{2}$ Y J. GARCIA-GUINEA ${ }^{3}$ \\ ${ }^{1}$ CIEMAT. Av. Complutense 22. Madrid 28040. España \\ ${ }^{2}$ Instituto de Cerámica y Vidrio (CSIC). Camino de Valdelatas s/n. Madrid 28049. España. \\ ${ }^{3}$ Museo Nac. CC. Naturales (CSIC). C/ José Gutiérrez Abascal 2. Madrid 28006. España.
}

\begin{abstract}
La leucita natural $\left(\mathrm{KAlSi}_{2} \mathrm{O}_{6}\right)$, materia prima para la fabricación de cerámicas dentales, es un material potencialmente válido para su utilización con fines dosimétricos ya que su respuesta luminiscente (radioluminiscencia -RL- y termoluminiscencia -TL-) en la región espectral del visible (200-800nm) es similar a la de otros aluminosilicatos utilizados también con fines dosimétricos. A pesar de la complejidad de las curvas, los espectros de emisión de TL y RL obtenidos con una leucita del Monte Somma (Nápoles, Italia) se pueden ajustar a un número máximo de seis funciones gaussianas situadas a 300, 380, 430, 480, 550 y $680 \mathrm{~nm}$ con significado físico. Este ajuste indica que en ambos procesos las trampas electrónicas que intervienen tienen el mismo origen. El análisis químico realizado por Fluorescencia de Rayos X (FRX) de la leucita estudiada permite intentar vincular el contenido de impurezas presentes en la red cristalina del material con las bandas de emisión que se producen como consecuencia de las distorsiones estructurales que se generan en la red cristalográfica formada por los tetraedros de $\mathrm{SiO}_{4}$ y $\left[\mathrm{AlO}_{4}\right]$.
\end{abstract}

Palabras clave: Leucita, radioluminiscencia, termoluminiscencia, espectro de emisión

\section{Radioluminescence and thermoluminescence emission spectra of a leucite of Monte Somma (Italy)}

Natural leucite $\left(\mathrm{KAlSi}_{2} \mathrm{O}_{6}\right)$, one of the main components of dental ceramics, shows similar radioluminescence (RL) and thermoluminescence (TL) response than other aluminosilicates (in the 200-800nm region) employed for dosimetric purposes. Despite the complex structure of the emission spectra, both glow curves can be fitted to six gaussian functions peaked at 300 , $380,430,480,550$ and $680 \mathrm{~nm}$. We infer that the involved electronic traps are of the same origin regardless of the luminescence process. The chemical analysis performed by X-ray fluorescence (XRF) allows us to seek relationships between the content of the impurities of the crystal lattice and the emission bands.

Keywords: Leucite, radioluminescence, thermoluminescence, emission spectra

\section{INTRODUCCIÓN}

El análisis de la emisión espectral de una fase mineral es de gran interés por los siguientes motivos: (i) Conocer mejor los procesos luminiscentes que tienen lugar en el material susceptible de ser utilizado como dosímetro. (ii) Adaptar el equipo de medida a las condiciones más ventajosas con el fin de obtener la información dosimétrica más precisa (i.e. selección adecuada de filtros y tubo fotomultiplicador). (iii) Detectar las modificaciones que sufren las bandas empleadas con fines dosimétricos cuando las muestras son tratadas térmicamente o están sometidas a exposiciones prolongadas a la luz solar, pues implicaría una estimación inexacta de la dosis. Con estos objetivos se han realizado medidas espectrales de termoluminiscencia (TL) y radioluminiscencia (RL) en una leucita natural $\left(\mathrm{KAlSi}_{2} \mathrm{O}_{6}\right)$ utilizada como materia prima para la elaboración de cerámicas dentales. La TL aporta información sobre las cargas (huecos y electrones) atrapadas que son liberadas tras el tratamiento térmico y sobre los centros de recombinación relacionados con defectos metaestables en el interior de la red (1). Por su parte, la RL, generada a partir de la excitación con partículas $(\alpha$ o $\beta$ ) o radiación (rayos $X$ o gamma) de alta energía, permite obtener información acerca de la eficiencia en la recombinación de electrones alojados en trampas superficiales y huecos atrapados en los centros luminiscentes. La RL es una técnica, que junto a catodoluminiscencia (CL) (2) y Resonancia Paramagnética de Espín (EPR) (3), se emplea en el estudio de defectos en minerales (4). Es habitual que los espectros de emisión de los materiales naturales posean una estructura muy compleja, sin embargo, hay un número limitado y específico de bandas que están asociadas a defectos cristalinos en la red, presencia de impurezas o cambios químicos (p. ej. reacciones redox); es decir, cada banda de emisión está asociada con una recombinación radiativa específica de cargas atrapadas $(5,6)$. Sin embargo, la interpretación de los espectros no es una tarea sencilla y requiere un estudio profundo de la muestra ya que por ejemplo, la luminiscencia debida a un ion depende de su posición en la red cristalina y del tipo de estructura en la que está alojado, es decir un mismo ion puede emitir en zonas diferentes. Un ejemplo típico es el $\mathrm{Mn}^{2+}$, si está tetraédricamente coordinado emite en el verde (550nm), pero si la coordinación es octaédrica la emisión aparece a una mayor longitud de onda, a 600nm (7). En este trabajo se muestran los resultados espectrales de TL y RL de un cristal de leucita en el que, empleando métodos matemáticos de deconvolución con gaussianas, se identifican cada una de las bandas que constituyen la curva. 


\section{MATERIALES Y MÉTODOS}

Se ha utilizado un cristal de leucita en forma de trapezoedro cúbico de $3 \mathrm{~cm}$, procedente del Monte Somma (Nápoles, Italia) (Fig. 1a). El análisis químico de la muestra se realizó por fluorescencia de rayos X (FRX) empleando un espectrómetro Phillips modelo PW-2424 con tubo de rayos X de ventana ultrafina y ánodo de rodio $(\mathrm{Rh})$ de alta potencia $2,4 \mathrm{~kW}$. La estructura cristalina de la leucita, analizada por difracción de rayos $X(D R X)$, se ajusta perfectamente a la leucita patrón de Villa Senni, Alban Hills, Italia (ficha ASTM-15-47), cuyas

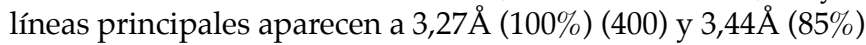
(004) comprobadas en un difractómetro automático Siemens D-5000 con radiación $\mathrm{CuK}_{\mathrm{A}}$ (Fig. 1b). La red cristalográfica tetragonal de la leucita (I4/a) a temperatura ambiente, se transforma en cúbica (Ia3d) cuando se calienta por encima de los $600^{\circ} \mathrm{C}$, aunque tiene un comportamiento reversible y en el enfriamiento hasta temperatura ambiente retorna a su red tetragonal $\left(\mathrm{I} 4_{1} / \mathrm{a}\right)$. La estructura cristalina de la leucita (Fig. 1c) se calculó utilizando el programa DIAMOND, a partir del refinamiento de parámetros PLV del difractograma y de las posiciones atómicas y los parámetros de la celdilla unidad $\left(a_{\mathrm{o}}=13.05476 ; b_{\mathrm{o}}=13.05476 ; \mathrm{c}_{\mathrm{o}}=13.75182 ; \alpha=\beta=\gamma=90^{\circ}\right)$ determinadas por Palmer et al., (8).

Las medidas de termoluminiscencia espectral de alta sensibilidad se han realizado en el espectrómetro de la Universidad de Sussex (U.K). Las señales se registraron en el rango 200nm-800nm y fueron corregidas para ajustarlas a la respuesta espectral del sistema. La irradiación de la muestra se llevó a cabo con un tubo de rayos X Philips MGMCN101

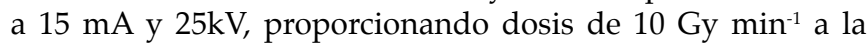
muestra (9).

El análisis espectral se efectuó utilizando en todos los casos fragmentos de dimensiones aproximadas $3 \times 3 \times 2.1 \mathrm{~mm}^{3}$ ( $\sim 5 \mathrm{mg})$ que fueron troceados cuidadosamente para evitar triboluminiscencia inducida (10). Las medidas de TL se efectuaron en atmósfera de $\mathrm{N}_{2}$ y a una velocidad de calentamiento de $5^{\circ} \mathrm{C} \cdot \mathrm{s}^{-1}$ hasta una temperatura máxima de $400^{\circ} \mathrm{C}$.

\section{RESULTADOS Y DISCUSIÓN}

\subsection{Caracterización de la muestra}

Los resultados obtenidos por DRX y FRX indican que la leucita analizada tiene un elevado grado de pureza. Su análisis químico $\left(\mathrm{Al}_{2} \mathrm{O}_{3}=22,9 \% ; \mathrm{SiO}_{2}=55,4 \% ; \mathrm{K}_{2} \mathrm{O}=18,4 \% ; \mathrm{Na}_{2} \mathrm{O}=1,17 \%\right.$; $\mathrm{Fe}_{2} \mathrm{O}_{3}=0,56 \% ; \quad \mathrm{CaO}=0,36 \% ; \quad \mathrm{RbO}_{2}=0,28 \% ; \quad \mathrm{P}_{2} \mathrm{O}_{5}=0,07 \%$; $\mathrm{MgO}=0,07 \% ； \mathrm{BaO}=0,064 \% ； \mathrm{TiO}_{2}=0,054 \% ； \mathrm{CsO}_{2}=0,045 \%$ ； $\mathrm{SO}_{3}=0,021 \% ; \mathrm{SrO}_{2}=0,017 \%$ y $\mathrm{MnO}=0,009 \%$ ) comparado con $\mathrm{su}$ composición teórica $\left(\mathrm{Al}_{2} \mathrm{O}_{3}=23,4 \%, \mathrm{SiO}_{2}=55,0 \%\right.$ y $\left.\mathrm{K}_{2} \mathrm{O}=21,6 \%\right)$ denota un menor contenido de $\mathrm{K}_{2} \mathrm{O}$ que se compensa con un mayor contenido de $\mathrm{Na}_{2} \mathrm{O}$. La apariencia externa de monocristal contrasta con la elevada saturación de maclado polisintético observado bajo microscopía de polarización con nicoles cruzados. Se aprecia un bajo grado de analcimización o alteración natural debido a un aumento del contenido en sodio y una disminución del contenido en $\mathrm{K}$, Ca y Mg. Los procesos de analcimización están asociados a rápidos enfriamientos de la lava basáltica encajante.

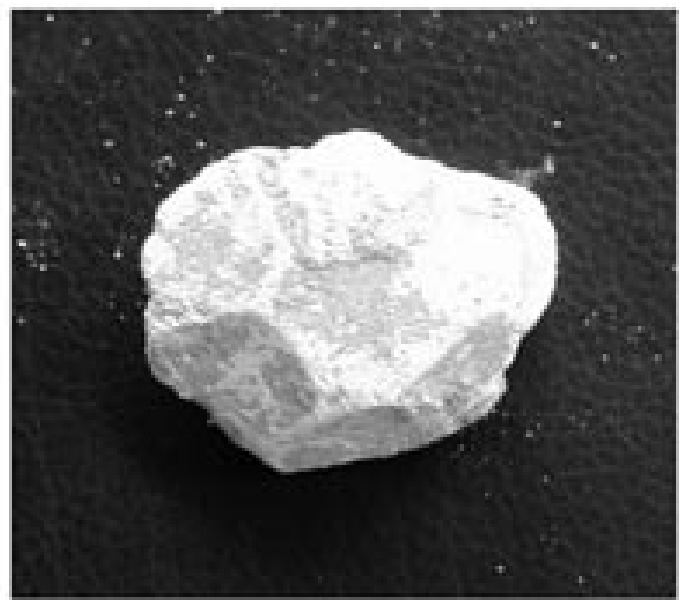

a)

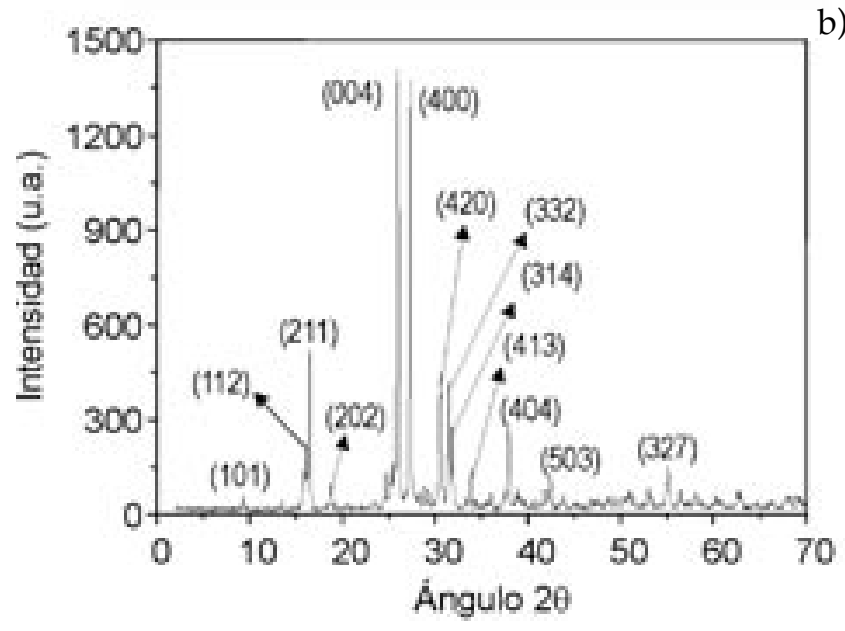

b)

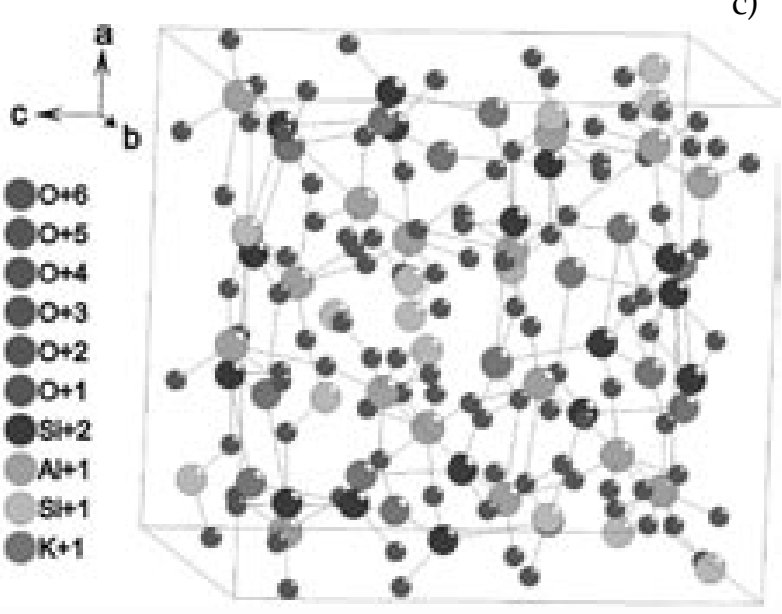

Figura 1. (a) Muestra de leucita procedente del Monte Somma (Italia) donde se observa su forma trapezoidal. (b) Difractograma de la muestra de leucita en polvo donde se detallan las líneas de difracción mas significativas. (c) Estructura de la leucita estimada a partir de los parámetros cristalográficos. 


\subsection{Espectros de emisión TL-RL. Asignación de bandas.}

Previamente a la obtención del espectro de TL, las muestras fueron irradiadas con una dosis total de 10Gy y almacenadas durante $48 \mathrm{~h}$ en presencia de luz roja. Los espectros TL y RL de cinco réplicas de cada una de las muestras, obtenidos en el rango de $200-800 \mathrm{~nm}$, fueron ajustados a un número nunca superior a seis funciones gaussianas utilizando el programa Peakfit (suministrado por Jandel Scientific Software). Todos los parámetros analizados que se presentan en la tabla 1 (posición del máximo, energía, FWHM, etc.) fueron estimados con un límite de confianza del $95 \%$; también se muestra el cálculo de la incertidumbre $(2 \sigma)$ asociada a cada medida. El criterio empleado para seleccionar el espectro de emisión de TL se basó en la posición donde el máximo es más intenso, a la temperatura de $200^{\circ} \mathrm{C}$ (Fig 2); la curva de RL se obtuvo a temperatura ambiente.

TABla 1. PARÁMETROS FÍSICOS OBTENIDOS A PARTIR DE LA DECONVOLUCIÓN CON FUNCIONES GAUSSIANAS DEL ESPECTRO DE EMISIÓN DE TL Y RL.

\begin{tabular}{|c|c|c|c|c|c|c|c|}
\hline \multicolumn{7}{|c|}{$\mathrm{N}^{\circ}$ de Pico } \\
\hline TL & 1 & 2 & 3 & 4 & 5 & 6 & $\mathrm{r}$ \\
\hline Posic.(nm) & $308 \pm 7$ & $383 \pm 9$ & $429 \pm 8$ & $476 \pm 9$ & $545 \pm 8$ & $696 \pm 12$ & \\
\hline E (eV) & $4,03 \pm 0,07$ & $3,24 \pm 0,08$ & $2,89 \pm 0,05$ & $2,61 \pm 0,05$ & $2,28 \pm 0,03$ & $1,78 \pm 0,03$ & \\
\hline Intensidad & $1,5 \pm 0,2$ & $7,4 \pm 0,5$ & $13 \pm 1$ & $14,5 \pm 0,5$ & $5,2 \pm 0,5$ & $6,5 \pm 0,5$ & \\
\hline Intensidad (\%) & $3,2 \pm 0,5$ & $15 \pm 1$ & $27 \pm 2$ & $30 \pm 5$ & $11 \pm 3$ & $13 \pm 1$ & 0,908 \\
\hline Area & $87 \pm 20$ & $511 \pm 28$ & $853 \pm 41$ & $1233 \pm 83$ & $459 \pm 42$ & $924 \pm 40$ & \\
\hline Area (\%) & $2,1 \pm 0,5$ & $12,6 \pm 0,7$ & $21 \pm 1$ & $30 \pm 2$ & $11 \pm 1$ & $23 \pm 1$ & \\
\hline FWHM & $53 \pm 3$ & $64 \pm 4$ & $61 \pm 4$ & $79 \pm 7$ & $83 \pm 7$ & $138 \pm 8$ & \\
\hline RL & & & & & & & \\
\hline Posic. (nm) & $304 \pm 4$ & $378 \pm 4$ & $430 \pm 4$ & $481 \pm 5$ & $567 \pm 10$ & $676 \pm 11$ & \\
\hline Energía (eV) & $4,07 \pm 0,05$ & $3,28 \pm 0,04$ & $2,88 \pm 0,03$ & $2,58 \pm 0,03$ & $2,19 \pm 0,04$ & $1,83 \pm 0,03$ & \\
\hline Intensidad & $0,62 \pm 0,04$ & $1,6 \pm 0,1$ & $3,4 \pm 0,3$ & $4,1 \pm 0,4$ & $2,2 \pm 0,3$ & $1,31 \pm 0,07$ & \\
\hline Intensidad(\%) & $4,3 \pm 0,3$ & $12 \pm 1$ & $26 \pm 2$ & $30 \pm 3$ & $17 \pm 2$ & $9,7 \pm 0,7$ & 0,931 \\
\hline Area & $22 \pm 4$ & $128 \pm 12$ & $232 \pm 12$ & $408 \pm 24$ & $206 \pm 24$ & $186 \pm 12$ & \\
\hline Area(\%) & $1,8 \pm 0,3$ & $11 \pm 1$ & $20 \pm 1$ & $34 \pm 2$ & $17 \pm 2$ & $16 \pm 1$ & \\
\hline FWHM & $36 \pm 3$ & $75 \pm 6$ & $64 \pm 4$ & $95 \pm 5$ & $87 \pm 6$ & $139 \pm 12$ & \\
\hline
\end{tabular}

La estimación razonable del número mínimo de picos válidos en la deconvolución de una curva, depende de varios factores. Una primera estimación consiste en comprobar, a simple vista, el número de cambios de pendiente que se aprecian en la curva lo que indica el número aproximado de máximos para ajustar la curva. Para confirmar esta primera apreciación, se recurre al siguiente razonamiento: (i) Aspectos matemáticos con el cálculo de la primera derivada y estimación del coeficiente de regresión $(r)$. (ii) Consideraciones físicas teniendo en cuenta los procesos que tienen lugar. Cuando las curvas son sencillas, los puntos de inflexión de las primeras derivadas se ajustan a cero y suministran información generalmente precisa sobre el número, posición y máxima anchura de los picos a media altura (FWHM, Full Width at Half Maximum), sin embargo, la complejidad de los espectros obtenidos de las muestras naturales dificulta esta labor ya que habitualmente hay varios picos solapados y los puntos de inflexión de las curvas no se ajustan a cero en la primera derivada. Por otra parte, y también desde un punto de vista matemático, el valor de $r$ puede mejorar algo añadiendo máximos de baja intensidad que sin

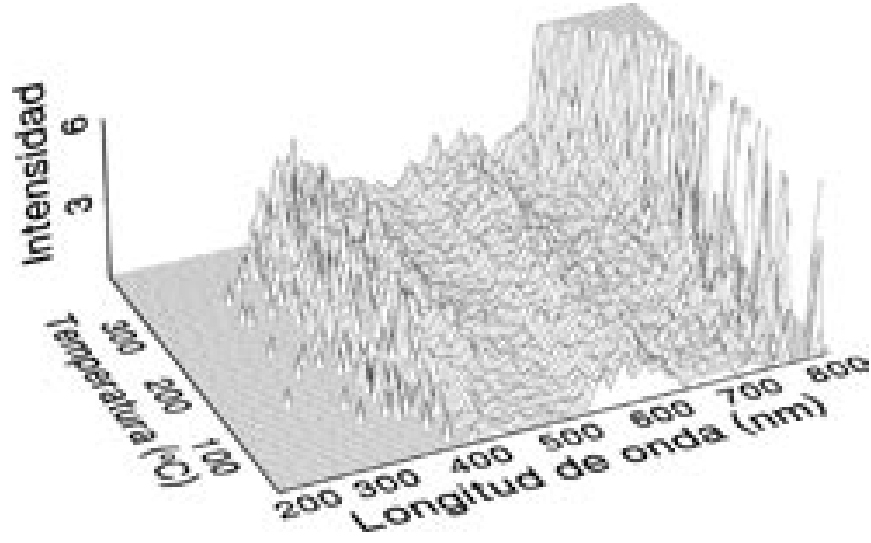

Figura 2. Diagrama tridimensional de termoluminiscencia de una muestra de leucita.

embargo no tienen un significado físico que permita correlacionarlos con defectos puntuales, cambios químicos, etc. Así pues, el número de bandas asignado que tenía un equilibrio entre las estimaciones matemáticas y el significado físico en la muestra leucita, nunca fue superior a seis, situados a 300, 380, 430, 470, 550 y 680nm (Tabla 1 y Fig 3).

La banda de $300 \mathrm{~nm}$ que aparece en la mayoría de los feldespatos y en muchos aluminosilicatos, parece estar relacionada con defectos asociados a la presencia de iones sodio en la red, normalmente exsoluciones de fases de feldespatos ricos en sodio. Algunos autores (11) han vinculado la aparición de esta banda con la presencia de $\mathrm{Pb}$ en la estructura cristalina. Sin embargo, se ha observado que en aluminosilicatos donde aparecía esta banda no se detectaba la presencia de $\mathrm{Pb}$ en $\mathrm{su}$ composición. Es más, Correcher y Garcia-Guinea (12) observaron en varios feldespatos ricos en $\mathrm{K}$ con estructuras similares, que la intensidad relativa de la banda de $300 \mathrm{~nm}$ aumentaba proporcionalmente con el contenido de Na determinado por análisis químico. En la leucita se observa que la intensidad relativa de esta banda es muy inferior a la observada en la albita (feldespato rico en sodio) medida en condiciones similares, donde aparece como la banda dominante en la región del UV (13). La posibilidad de utilizar esta banda con fines dosimétricos (principalmente en datación) ha sido desestimada por los siguientes motivos: (i) Los lectores de luminiscencia convencionales están equipados con fotomultiplicadores protegidos por una ventana de cuarzo que no permite el paso de la emisión UV. (ii) La emisión UV podría generar fototransferencia de electrones de trampas más profundas a más superficiales (14) lo que supone un incremento en el número de cargas procedente de esta emisión luminiscente. (iii) Los procedimientos de precalentamiento convencional utilizados en datación pueden no ser suficientes para eliminar esta contribución. Sin embargo, y a pesar de estos inconvenientes, se han realizado algunos estudios en albita observando que el comportamiento de esta banda de emisión podría ser usada con fines dosimétricos (13). La presencia de esta banda en los aluminosilicatos potásicos permitiría potencialmente su utilización en dosimetría, aunque su baja intensidad relativa es un impedimento para su aplicación en este campo.

La emisión UV-azul centrada en $380 \mathrm{~nm}$ es una banda característica de las fases minerales que contienen tetraedros $\mathrm{SiO}_{4}$ (cuarzo y silicatos) estando asociada a la existencia de 
Intensidad (u.a.)

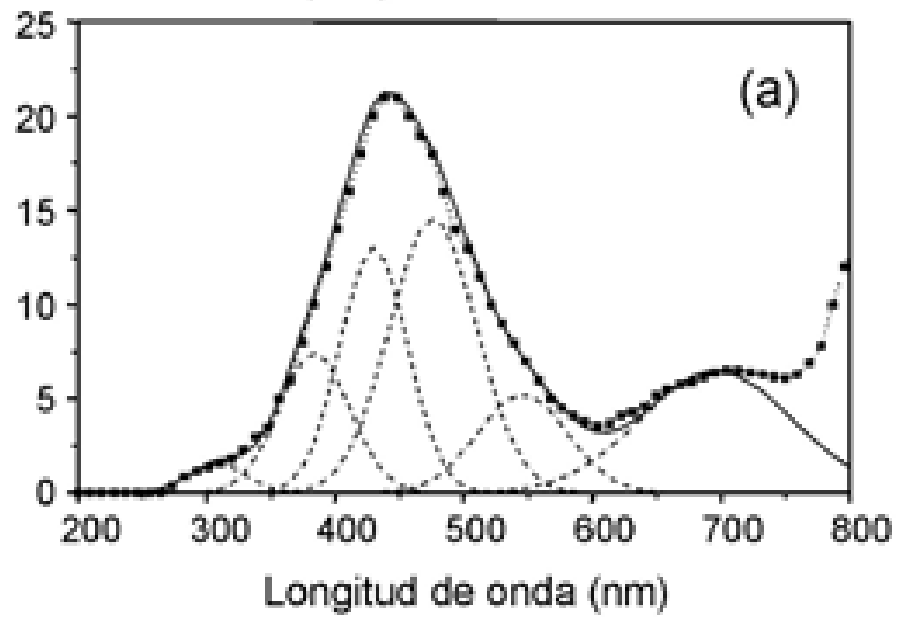

$\mathrm{dl} / \mathrm{d} \lambda$

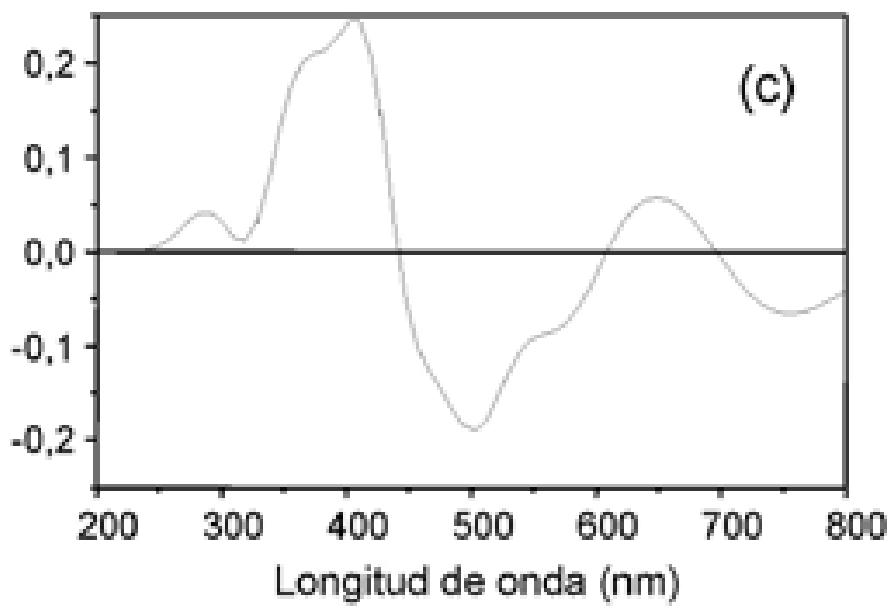

Intensidad (u.a.)

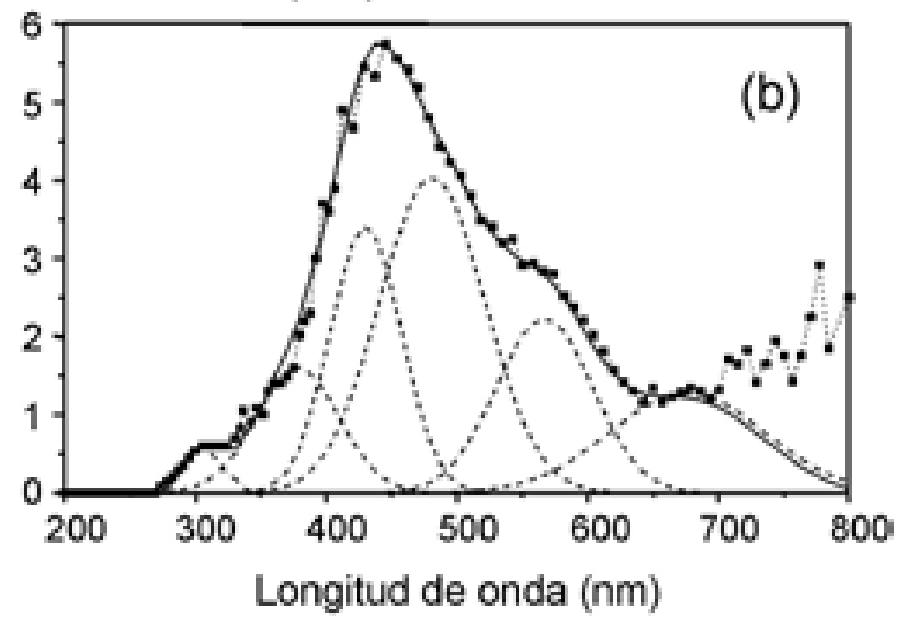

$\mathrm{d} / \mathrm{d} \lambda$

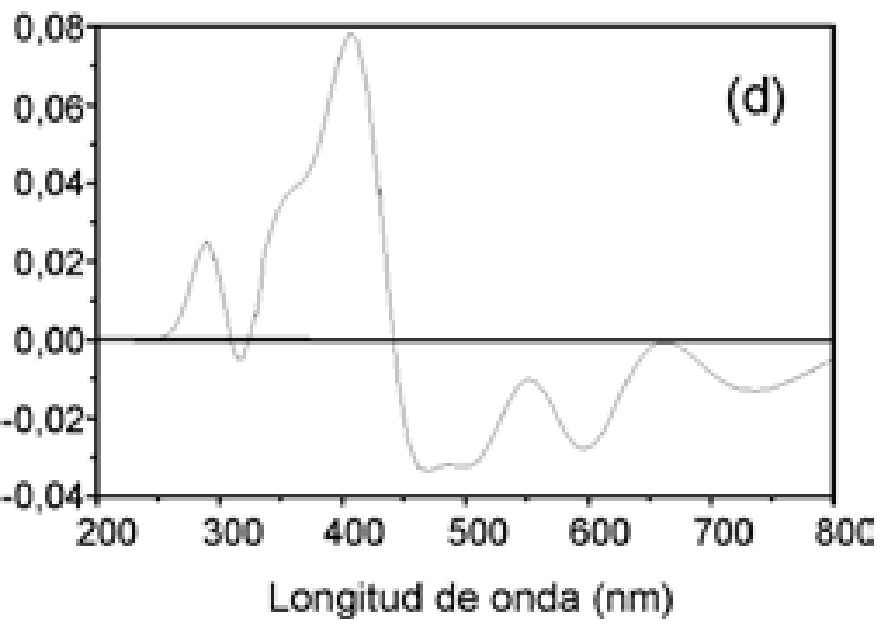

Figura 3. Deconvolución de los espectros de emisión de (a) TL a $200^{\circ} \mathrm{C}$ y (b) RL de leucita. (c) corresponde al cálculo de la primera derivada del espectro de TL y (d) al espectro de RL.

defectos intrínsecos de la red cristalina producidos por la presencia de iones alcalinos intersticiales situados en posiciones adyacentes a átomos de $\mathrm{Al}^{3+}$ (15). Esta banda es muy sensible a la radiación por lo que es la que mayoritariamente se emplea tanto en datación como en dosimetría retrospectiva. La radiación suministrada produce una migración de álcalis que generan un alto número de pares electrón-hueco en la red; algunos de estos huecos pueden ser capturados formándose centros $\left[\mathrm{AlO}_{4} / \mathrm{M}^{+}\right]$. Cuando la energía suministrada es suficiente, se produce una recombinación de electrones con los huecos atrapados en posiciones adyacentes a $\mathrm{Al} / \mathrm{M}^{+}$que reduce la presencia de los compensadores de carga induciendo la emisión luminiscente de $380 \mathrm{~nm}$ en los centros $\left[\mathrm{AlO}_{4}\right]^{\circ}$. Martini et al (16) han descrito un efecto similar en el cuarzo. Como se aprecia en la figura 3 este máximo, típico de otros aluminosilicatos potásicos (12), presenta valores estrechos de FWHM.

El origen de la banda de emisión de 430nm no está bien definido. Algunos autores (17) asumen que se genera como consecuencia de la presencia de iones $\mathrm{Cu}$ (II) que sustituyen a cationes divalentes $\left(\mathrm{Ca}^{2+}\right)$ y que están situados próximos a depósitos de huecos. Otros autores (11) atribuyen esta emisión, que aparece en la mayoría de los aluminosilicatos con composiciones químicas diferentes y orden variable de $\mathrm{Al}$ y $\mathrm{Si}$, a la recombinación que se produce en un centro vinculado a defectos reticulares del tipo $\mathrm{Al}-\mathrm{O}-\mathrm{Al}$ o Si-O-Si. La aparición de esta banda en la leucita no podría asociarse con la presencia de $\mathrm{Cu}^{2+}$ (no ha sido detectado por FRX) con lo que parece más acertado pensar que se debe a esta segunda posibilidad; no obstante, es necesario insistir sobre el tema para determinar el origen de esta banda. La banda que aparece a $470 \mathrm{~nm}$ podría estar asociada a la presencia de $\mathrm{Ti}^{4+}(0,054 \%)$. Este catión se incorpora fácilmente en la red del aluminosilicato ocupando posiciones tetraédricas en sustitución de $\mathrm{Si}^{4+}$ (15). Las bandas de 430 y $470 \mathrm{~nm}$ aparecen en la mayoría de los aluminosilicatos, sin embargo presentan dos inconvenientes importantes para su utilización con fines dosimétricos: (i) Son bandas que aparecen muy solapadas entre ellas y tienen una contribución importante de la emisión del verde, lo que implica que no existen filtros adecuados para la discriminación espectral y (ii) Su intensidad relativa es muy variable lo que sugiere baja estabilidad. 
La banda en la región del verde, centrada en 550nm, aparece en la casi totalidad de los aluminosilicatos alcalinos y su origen se atribuye a la presencia de iones $\mathrm{Mn}^{2+}$ en la estructura cristalina en posiciones tetraédricas habitualmente ocupadas por $\mathrm{Ca}^{2+}(18)$. En general existe una mayor abundancia de $\mathrm{Mn}^{2+}$ en plagioclasas que en feldespatos ricos en potasio y esto es debido a que los radios iónicos de $\mathrm{Mn}(0,80), \mathrm{Na}(0,97)$ y Ca $(0,99)$ no existe una variación importante entre ellos, aunque en comparación con el K (1.33) hay una considerable diferencia. En la leucita se aprecia la presencia de esta banda y en los análisis de FRX se detectó un contenido de $\mathrm{Mn}^{2+}$ del $0,009 \%$.

Por último, la banda que aparece a menor energía, a $680 \mathrm{~nm}$, se asocia a la presencia de $\mathrm{Fe}^{3+}$ en la red cristalina de los aluminosilicatos. Este catión sustituye a átomos de Si o $\mathrm{Al}$ en posiciones $\mathrm{T}$ actuando como centro de recombinación para huecos o electrones según sea su valencia. Esta emisión se genera como consecuencia de la incidencia de la radiación con el ion dando lugar a un proceso redox $(5,19)$. Aunque se han hecho intentos de emplear esta banda con fines dosimétricos, se ha desestimado su utilización debido a la inestabilidad detectada en esta señal (20). Finch y Klein (21), utilizando diferentes técnicas (espectroscopia por catodoluminiscencia -CL-, espectrometría de masas de iones secundarios -SIMS- y EPR), corroboraron que esta emisión era debida a la presencia de $\mathrm{Fe}^{3+}$. Observaron que cuando el hierro estaba situado en una posición tetraédrica $\left(\mathrm{T}_{1}\right)$, en feldespatos ordenados, la banda aparecía en el rojo, a menor longitud de onda que si el catión estaba alojado en un feldespato con menor triclinicidad (mayor desorden); en esta situación la banda de emisión se detectaba en el infrarrojo. Es decir, en función del sistema cristalino donde este catión esté alojado la posición de la banda de emisión varía. Este hecho, unido a que se trata de la banda más ancha (tabla 1), hace que precisión y exactitud de la medida sea demasiado compleja. Por otra parte, Kirsh y Townsend (5) determinaron que parte de la emisión TL en el rojo era una 'emisión secundaria' de la TL azul generada por la absorción de fotones azules por parte de los $\mathrm{Fe}^{3+}$, los cuales son re-emitidos como luz roja. Otra característica que hace inviable la utilización de esta banda con fines dosimétricos es la recombinación radiativa que se produce por efecto túnel y que está íntimamente ligada al decaimiento de señal con el tiempo anómalo (22). Por este motivo, no se puede determinar el error cometido en la estimación de la dosis.

Con independencia de observar que en el proceso luminiscente que tiene lugar no se superan las seis bandas, es importante destacar otros dos aspectos: (i) Los coeficientes de regresión (r) que indican la calidad del ajuste obtenidos han sido muy aceptables independientemente del proceso luminiscente que tiene lugar. (ii) La desviación standard $(2 \sigma)$ de los valores de energía de los picos no fue nunca superior al $4 \%$.

\section{CONCLUSIONES}

Tanto en TL como en RL, las trampas electrónicas que intervienen en los procesos tienen el mismo origen, no habiendo diferencias importantes debidas a la estructura cristalina del material. Aparentemente, la variación estructural influye en la intensidad de las bandas, pero no en su posición cuya variación nunca fue superior a un $4 \%$. A pesar de la complejidad de los espectros, en todo los casos se pudieron ajustar las curvas a un número máximo de seis funciones gaussianas situadas en 300, 380, 430, 480, 550 y 680nm. La aparición de estas bandas de emisión es principalmente debida a la presencia de impurezas $\left(\mathrm{Na}^{+}, \mathrm{Mn}^{2+}, \mathrm{Fe}^{3+}\right.$, etc.) que provocan distorsiones estructurales en la red cristalográfica formada por tetraedros de $\mathrm{SiO}_{4}$ y $\left[\mathrm{AlO}_{4}\right]$. En esta situación, una información añadida que ofrece el espectro de emisión es la determinación cualitativa de la composición química. Por otra parte, el análisis químico de la leucita indicará la presencia de bandas determinadas que será de utilidad para la elección del sistema de detección apropiado (filtros y tubos fotomultiplicadores) al estudiar aluminosilicatos para su empleo con fines dosimétricos donde la emisión a 380nm parece ser la más apropiada para datación o dosimetría retrospectiva.

\section{AGRADECIMIENTOS}

Este trabajo ha sido financiado por la CICYT (proyecto BFM2002-00048). Los espectros de TL y RL se realizaron en la Univ. de Sussex (UK) con la ayuda del Prof. P.D.Townsend.

\section{REFERENCIAS}

1. S.W.S. McKeever. "Thermoluminescence of solids". Cambridge Univ. Press, Cambridge, 1985.

2. M.A.S. Kalceff, G.J. Thorogood y K.T. "Short. Charge trapping and defect segregation in quartz". J. Appl. Phys. 86[1] 205-208 (1999).

3. B. Speit y G. Lehmann. "Hole centers in the feldspar sanidine". PhysicaStatus-Solidi-A. 36[2] 471-481 (1976).

4. T. Trautmann, M.R. Krbetschek, A. Dietrich y W. Stolz. "Feldspar radioluminescence: a new dating method and its physical background". J. Lumin. 85[1-3] 45-58 (1999).

5. Y. Kirsh y P.D. Townsend. "Speculations on the blue and red bands in the TL emission spectrum of albite and microcline". Nucl. Tracks. Radiat. Meas. 14[1-2] 43-49 (1988).

6. J. García-Guinea, H.M. Rendell y L. Sanchez-Muñoz. “Luminescence spectra of alkali feldspars: some relationships between structural features and luminescence emission". Radiat. Prot. Dosim. 66[1-4] 395-399 (1996).

7. A. Vandie A.C.H.I. Leenaers, W.F. Vanderweg y G. Blasse. “A search for luminescence of the trivalent manganese ion in solid aluminates". Mater. Res. Bull. 22[6] 781-788 (1987).

8. D.C. Palmer, M.T. Dove, R.M. Ibberson y B.M. Powell. "Structural behavior, crystal chemistry, and phase transitions in substituted leucite: High-resolution neutron powder diffraction studies". Am. Mineral. 82[1-2] 16-30 (1997).

9. B.J. Luff y P.D. Townsend. "High sensitivity thermoluminescence spectrometer". Meas. Sci. Technol. 4[10] 65-71(1993).

10. J. Garcia-Guinea y V. Correcher. "Luminescence spectra of alkali feldspars: Influence of crushing on the ultraviolet emission band" Spectrosc. Lett., 33 103-113 (2000).

11. A. Speit y G. A. Lehmann. "Comparative study of thermoluminescence and isothermal destruction of radiation defects in feldspars" J. Lumin., 27 127-136 (1982)

12. V. Correcher y J. García-Guinea. "On the luminescence properties of adularia feldspar". J.Lumin. 93[4] 303-312 (2001)

13. V. Correcher, J.M. Gomez-Ros y A. Delgado. "The use of albite as a dosemeter in accident dose reconstruction". Radiat. Prot. Dosim. 84[1-4] 547-549 (1999).

14. I.K. Bailiff y N.R.J. Poolton. "Studies of charge transfer mechanism in feldspars". Nucl. Tracks Radiat. Meas. 18 111-118 (1991).

15. M.L. Clarke y H.M. Rendell. “Thermoluminescence, radioluminescen- 
ce and cathodoluminescence spectra of alkali feldspars". Radiat. Meas. 27[2] 263-272 (1997).

16. M. Martini, A. Paleari, G. Spinolo y A. Vedda. Role of $\left[\mathrm{AlO}_{4}\right]^{\circ}$ centers in the $380 \mathrm{~nm}$ thermoluminescence of quartz". Phys. Rev. B 52[1] 138-142 (1995).

17. H.M. Rendell, M.R. Khanlary, P.D. Townsend, T. Calderón y B.J. Luff. "Thermoluminescence spectra of minerals". Miner. Magaz. 57 217-222 (1993).

18. J.E. Geake, G. Walker, D.J. Telfer, A.H. Mills y G.F.J. Garlick. “Luminescence of lunar, terrestrial and synthesized plagioclase caused by $\mathrm{Mn}^{2+}$ and $\mathrm{Fe}^{3+\prime}$. Geochim. Cosmochim. Ac. 3 3181-3189 (1973).
19. D.J. Telfer y G. Walker, G. "Optical detection of $\mathrm{Fe}^{3+}$ in lunar plagioclase". Nature. 258 694-695 (1975).

20. A.J.C. Zink y R. Visocekas. "Datability of sanidine feldspar using the near-infrared TL emission". Radiat. Meas. 27 251-261 (1997).

21. A.A. Finch y J. Klein. "The causes and petrological significance of cathodoluminescence emissions from alkali feldspars". Contrib. Mineral. Petr. 135[2/3] 234-243 (1999).

22. R. Visocekas, N.A. Spooner, A. Zink y P. Blanc. "Tunnel afterglow, fading and infrared emission in thermoluminescence of feldspar". Radiat. Meas. 23 377-385 (1994).

Recibido: 03.11 .03

Aceptado: 14.03 .04 